\title{
Estado actual del ultrasonido de alta frecuencia (HIFU) en el tratamiento del adenocarcinoma prostático
}

\author{
Lledó García E, Jara Rascón J, Herranz Amo F, Hernández Fernández C. \\ Servicio de Urología. Hospital General Universitario Gregorio Marañón. Madrid.
}

Actas Urol Esp. 2007;31(6):642-650

\section{RESUMEN}

\section{ESTADO ACTUAL DEL ULTRASONIDO DE ALTA FRECUENCIA (HIFU) EN EL TRATAMIENTO DEL ADENOCARCINOMA PROSTÁTICO}

Objetivo. Evaluar el estado actual de la utilización del Ultrasonido de Alta Intensidad (HIFU) en el tratamiento del adenocarcinoma prostático (Cap).

Método. Hemos llevado a cabo una revisión del papel actual del HIFU en el tratamiento del Cap.

Resultados. La tecnologia HIFU está actualmente siendo aplicada ya de forma habitual en Europa en el tratamiento del Cap. Las indicaciones principales en la actualidad son dos: la aplicación del HIFU como primer escalón terapéutico y como terapia de rescate en recidivas post-radioterapia. Los trabajos actuales están basados en equipos clínicos en desarrollo. Aunque las últimas publicaciones sugieren que HIFU es una forma de tratamiento útil para el Cap bien y moderadamente diferenciado, existe todavía falta de comparaciones de esta terapia menos invasiva con los tratamientos estándar. Seguimos detectando en los trabajos disparidad en la definición de supervivencia libre de enfermedad (SLE), lo que dificulta la interpretación de resultados y la extracción de conclusiones definitivas.

Conclusiones. La experiencia de los grupos de trabajo especializados en HIFU es altamente prometedora. Entre los aspectos a destacar está su gran capacidad de destrucción tumoral local tanto en los casos primarios como en las recidivas post-radioterapia. Para extraer conclusiones a medio y largo plazo debemos generar ensayos clínicos randomizados y controlados con seguimiento suficiente para medir beneficios en términos de supervivencia global y calidad de vida (balance efectos adversos / beneficios), realizar comparaciones con las terapias estándar y homogeneizar los criterios de definición de la SLE.

Palabras clave: Ultrasonido de alta frecuencia (HIFU). Adenocarcinoma prostático.

\section{ABSTRACT \\ CURRENT STATE OF HIGH INTENSITY FOCUSED ULTRASOUND (HIFU) AS TREATMENT OF PROSTATIC CARCINOMA}

Objective. To evaluate the current state of high intensity focused ultrasound as therapeutical option of prostatic carcinoma $(\mathrm{PCa})$

Methods. We completed an extense review of urologic literature on the role of HIFU on the treatment of PCa.

Results. This technique is nowadays usually being indicated in Europe as treatment of many cases of either primary or relapsed PCa after radiotherapy. Although some reports suggest that HIFU is very effective as treatment for low and medium risk localized PCa patients, no randomized series comparing this technique with conventional therapies have been presented yet. Great disparity in criteria to define free-disease survival is detected, which make difficult the interpretation of results.

Conclusions. Experience of some groups in HIFU is highly promising. Local tumour destruction is evident both in primary and relapsed PCa cases. To make conclusions in the long-term, controlled-randomized trials must be designed, with follow-up to measure benefits in global survival and quality of live. Comparisons must be completed with conventional techniques, and a uniform definition of disease free-survival is necessary. 


\section{INTRODUCCIÓN Y ANTECEDENTES}

El adenocarcinoma prostático es la neoplasia más frecuentemente diagnosticada en los varones de USA $^{1}$ y el segundo tumor más frecuente en Gran Bretaña y Gales $^{2}$. En un estudio reciente sobre la incidencia del Cap en la Comunidad de Madrid, la incidencia estimada de esta neoplasia ha sido calculada en 100.4 casos/100.000 hombres $^{3}$. El antígeno prostático específico (PSA) y sus diversas formas moleculares, que permiten una sospecha de la presencia del Cap en fases asintomáticas, pemiten la detección de esta enfermedad en fases de completa curabilidad.

El tratamiento convencional del Cap localizado incluye la actitud de vigilancia expectante, la prostatectomía radical (retropúbica abierta o laparoscópica, perineal) y la radioterapia ${ }^{2}$. Todas estas opciones pueden producir efectos secundarios no deseados, como la incontinencia urinaria y la disfunción eréctil. En los últimos años se están desarrollando una serie de tratamientos que combinan menor invasividad -con todas las ventajas que este concepto supone para el paciente- con aparente efectividad oncológica y reducción de los efectos secundarios. Técnicas que perfeccionan la radioterapia (braquiterapia, radioterapia conformacional $3 \mathrm{D}$ ), innovaciones tecnológicas en crioterapia, ultrasonidos de alta intensidad (HIFU). Otras alternativas, como la terapia génica, se encuentran en fases completamente precoces de su desarrollo.

El ultrasonido de alta intensidad (HIFU) basa su efecto en la inducción de necrosis coagulativa en cualquier medio no viscoelástico, como los tejidos biológicos ${ }^{1}$. Las ondas focalizadas de ultrasonido son emitidas desde un transductor y absorbidas en un área objetivo, produciendo efectos mecánicos, cavitación y efectos térmicos (intensa elevación térmica local) sin lesionar los tejidos que se encuentran interpuestos entre el emisor y el objetivo, en el camino del ultrasonido ${ }^{4}$. El potencial efecto antitumoral del HIFU fue ya mostrado en el terreno experimental por Chapelon ${ }^{5}$, posteriormente también en el terreno clínico ${ }^{6}$.

\section{MECANISMO DE ACCIÓN DEL HIFU}

La elevación de la temperatura tisular depende del coeficiente de absorción de los tejidos (medida de energía acústica convertida en calor en los tejidos), del tamaño y forma del órgano, así como de la respuesta térmica de la zona calentada (Fig. 1). Los cambios biológicos inducidos por el calor dependen de la temperatura alcanzada y de la duración de la exposición. Para temperaturas por encima de un determinado valor se produce un daño tisular irreversible, en forma de necrosis coagulativa. Para dosis térmicas (considerando dosis térmica como el tiempo equivalente en segundos de exposición a una temperatura estándar de $\left.43^{\circ} \mathrm{C}\right)$ por debajo de un cierto umbral, el efecto depende de la sensibilidad tisular al calor ${ }^{7}$.

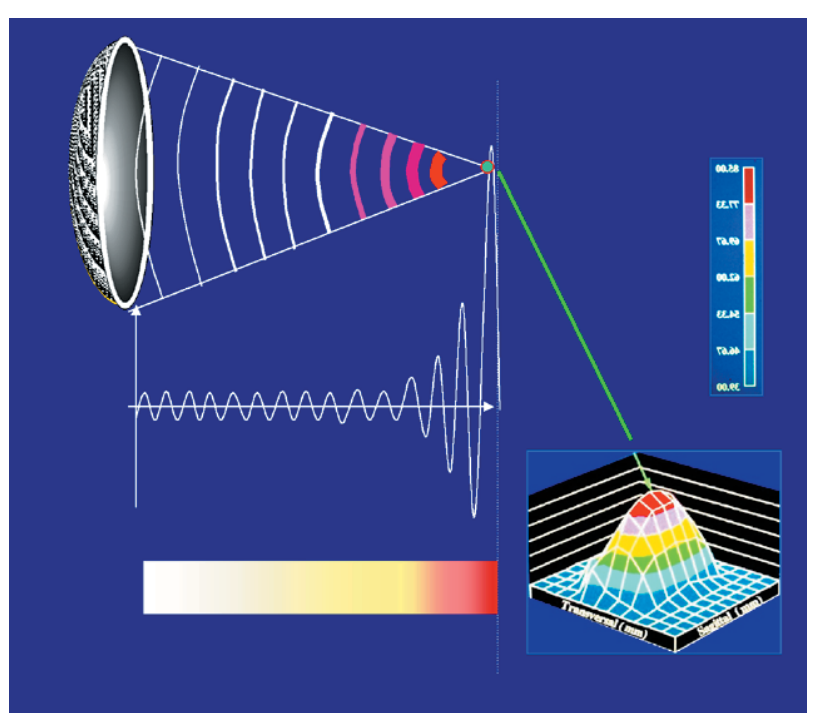

FIGURA 1. Principio de acción de HIFU: la generación de ondas ultrasónicas focalizadas produce un incremento de densidad energética en el área focal sin modificaciones en el tejido penetrado.

HIFU está siendo probado en varias potenciales aplicaciones urológicas ${ }^{8,9}$. El haz energético generado por el dispositivo de ultrasonido de alta frecuencia es muy intenso en la denominada zona focal, con una concomitante dramática disminución de esa misma temperatura, de manera simultánea, en los tejidos que rodean al punto crítico. Se produce un rápido incremento de la temperatura de forma focalizada (por encima de valores de $70-100^{\circ} \mathrm{C}$ ) en pocos segundos, destruyendo una zona de tejido muy específico, con absoluta conservación de la estructura tisular circundante. El volumen de tejido destruido por un solo disparo energético, se denomina lesión elemental. Para conseguir una destrución progre- 
siva del órgano se producen varias y sucesivas lesiones elementales mediante un desplazamiento mecánico del transductor o bien a través de un posicionamiento electrónico del punto focal, cuando se dispone de esta opción en el dispositivo utilizado.

El dispositivo o transductor de terapia ultrasónica está caracterizado por algunos aspectos:

- Número de oscilaciones completas/segundo de la longitud de onda generada por el transductor.

- Materiales piezoeléctricos del dispositivo (cristales que experimentan una deformación mecánica cuando se ven sometidos a un potencial eléctrico): los más modernos pueden actuar a la suficiente densidad de potencia (concepto que cuantifica la potencia por unidad de área normal en la dirección de propagación) de forma que aseguren estabilidad consistente a largo plazo para conseguir los efectos que se pretenden de la terapia. Estos materiales permiten, además, el diseño de formas geométricas adaptables a las diversas restricciones anatómicas. El tratamiento actual con HIFU de volúmenes mayores de tejido se consigue mediante el desplazamiento mecánico del transductor (dispositivos de un solo foco): las aplicaciones futuras suponen la utilización de transductores que permitan desplazar el punto focal sin mover el dispositivo ${ }^{7}$.

- Parámetros sónicos: intensidad acústica (potencia acústica media transmitida por unidad de área); duración de la exposición (tiempo durante el cual la onda de ultrasonido es aplicada al tejido en cada disparo de HIFU); proporción on-off (relación entre la duración de la exposición del tejido y el tiempo de espera entre disparos de HIFU).

\section{EXPERIENCIAS EN MODELOS ANIMALES}

Los modelos de cáncer experimental utilizados para evaluar el HIFU han sido múltiples. Desde modelos in vivo (glioma murino, meduloblastoma, hepatoma) ${ }^{10-12}$. Los modelos en Cap han sido llevados a cabo utilizando líneas celulares DUNNING R3327 implantados en ratas, sublíneas de cáncer AT2 y AT6, con alto potencial metastási$\mathrm{co}^{13}$. Los modelos animales han sido aplicados a tumores renales ${ }^{14}$, evidenciando posibilidades de destrucción efectiva local sin producción de metástasis. La administración por vía transrectal de HIFU ha sido experimentada en perros para su aplicación en próstata ${ }^{15}$.

\section{EXPERIENCIAS CLÍNICAS EN TUMORES NO PROSTÁTICOS}

HIFU ha sido aplicado en la destrucción de tumores vesicales superficiales ${ }^{16}$ aunque con resultados poco competitivos al compararlos con el tratamiento clásico endoscópico. El cáncer renal se encuentra actualmente, tras una fase de estudios experimentales, en desarrollo de investigación clínica. Algunos estudios piloto han evidenciado necrosis de volúmenes tisulares, con reducción progresiva del tamaño tumoral tras el tratamiento ${ }^{17}$.

\section{APLICACIÓN EN EL CÁNCER PROSTÁTICO}

\section{Tipos de dispositivos}

Existen en la actualidad 2 tipos de dispositivos (Fig. 2):

\section{Dispositivo Ablatherm ${ }^{R}$}

La máquina dispone de un módulo de tratamiento que incluye la camilla del paciente, el sistema de posicionamiento de la sonda, el sistema de enfriamiento para la conservación de la temperatura de la pared rectal y el transductor ultrasónico utilizado durante la fase de localización anatómica. Se dispone de una sonda endorectal de tratamiento/imagen que trabaja a $7.5 \mathrm{MHz}$ y de un transductor de tratamiento, focalizado a $40 \mathrm{~mm}$ que trabaja a $3 \mathrm{MHz}$. Este sistema incorpora múltiples mecanismos de seguridad: estabilización de la pared rectal durante los movimientos del transductor, control permanente de la distancia entre transductor y pared rectal y detector de movimientos del paciente que paraliza el sistema si estos movimientos se producen durante la fase de disparo. Los parámetros de tratamiento son seleccionados para conseguir aumentar el tamaño de la lesión generada, manteniendo la indemnidad de la pared rectal y tejidos circundantes. El tamaño de la lesión elemental está entre 19 y $24 \mathrm{~mm}$ de longitud y 1,7 mm de diámetro. Según las características del paciente se modificarán los parámetros definitivos: pulsos de tratamiento de 5 segundos con intervalos entre disparos de 5 segundos para 
la primera sesión en casos primarios; pulsos de 4,5 segundos con intervalos de 5 segundos para sesiones sucesivas en casos primarios; pulsos de 4 segundos con intervalos de 7 segundos en casos de recidiva local tras radioterapia.

Los tratamientos son llevados a cabo bajo anestesia general, en posición lateral. La sonda endorectal es insertada en un balón de látex rellenado con líquido de enfriamiento e introducido en el recto. Tras definir los límites del volumen objetivo del tratamiento por el operador, el tratamiento se realiza desde el ápex a la base de la glándula prostática. Habitualmente se definen 4-8 volúmenes para tratar toda la glándula. Al finalizar el tratamiento se coloca un catéter uretral o suprapúbico.

\section{Dispositivo Sonoblate ${ }^{R}$}

No posee cama-específica para el tratamiento. Se dispone de varias sondas, seleccionadas por el operador de acuerdo al tamaño de la lesión elemental requerida: $10 \mathrm{~mm}$ de longitud y $2 \mathrm{~mm}$ de diámetro (longitud de onda de $25 \mathrm{~mm}$ o $45 \mathrm{~mm}$ ); $10 \mathrm{~mm}$ de longitud y $3 \mathrm{~mm}$ de diámetro (longitud de onda de 30-35 ó $40 \mathrm{~mm}$ ). La sonda es elegida según el tamaño de la glándula prostática a tratar: las glándulas más grandes requieren longitudes de onda mayores. El tratamiento es usualmente realizado bajo anestesia general, en 3 cortes coronales, empezando por la parte anterior de la próstata y desplazándose hacia la zona posterior. Durante el tratamiento, usualmente, se realiza al menos un cambio de sonda.

\section{Resultados de la técnica. Escenarios clínicos actuales}

Las experiencias más desarrolladas actualmente se refieren al Cap. Hemos seleccionado una serie de trabajos basándonos en el número de casos o de una cierta solidez/homogeneidad en los criterios de inclusión de los pacientes, así como en el tiempo de seguimiento y la aplicación de un criterio definido de supervivencia libre de enfermedad. El mayor número de estudios correspondieron a series descriptivas de casos. Ningún ensayo clínico randomizado pudo ser evaluado.

El análisis de resultados sobre la eficacia clínica debe valorar, especialmente, la existencia de ensayos clínicos randomizados. Las series descriptivas de casos muestran sesgos referidos a los cri- terios de selección de los pacientes y a la evaluación de los objetivos, con seguimientos cortos. En la actualidad no se han realizado aún comparaciones del HIFU con los tratamientos estándar. Tampoco se han publicado estudios sobre el costeefectividad de esta técnica. Según $\mathrm{Hummel}^{2}$ de un listado de 15 posibles terapias emergentes, el HIFU se encontraría en el mismo grupo de evidencias que RITA, terapia génica o la fotocoagulación (Fig. 3).

Un importante problema con el que nos encontramos al revisar la literatura es la ausencia de criterios homogéneos para definir el concepto de supervivencia libre de enfermedad (SLE). Este aspecto es fundamental para valorar los resultados obtenidos por los distintos grupos de trabajo. Según Critz ${ }^{18}$ el análisis multivariante de factores relacionados con ausencia de enfermedad revelan que la definición de estatus libre de enfermedad utilizada para calcular la SLE es tan significativa como el PSA pre-tratamiento o el Gleason medio (Fig. 4). Detectamos hasta 7 puntos de corte distintos de PSA publicados en los últimos años por autores de primera línea ${ }^{1,18-20}$. Esto supone un grave problema en dos aspectos: en primer lugar, la interpretación del resultado de los seguimientos. Por otro lado, el significado clínico real de la progresión bioquímica del Cap tras un tratamiento radical. Hasta un $26 \%$ de prostatectomías radicales pueden dejar restos de tejido glandular benigno en los márgenes quirúrgicos, lo que explicaría, al menos, una gran parte de los casos de progresión de PSA hasta valores $</=0,3 \mathrm{ngrs} / \mathrm{ml}$ sin cambios en períodos de 3 años desde su detección ${ }^{21}$.

Analizamos en primer lugar la indicación de HIFU como primera terapia. Debemos destacar 2 artículos: el primero de ellos es el Estudio Multicéntrico Europeo publicado en Urology en 2004 dirigido por Thüroff ${ }^{19}$. Se trata de un ensayo clínico fase II-III prospectivo, que evalúa la seguridad y eficacia de HIFU en el tratamiento del Cap localizado (T1-2, NO-X, MO) en pacientes no candidatos a prostatectomía radical por problemas médicos o por no deseo de someterse a los riesgos de la intervención quirúrgica. Los pacientes $(\mathrm{N}=402)$ fueron incluidos entre 1995 y 1999 en seis grandes centros europeos. La edad media fue de $69,3 \pm 7,1$ años. El volumen prostático medio era de $28 \pm 13,8$ cc y la concentración media de PSA de 

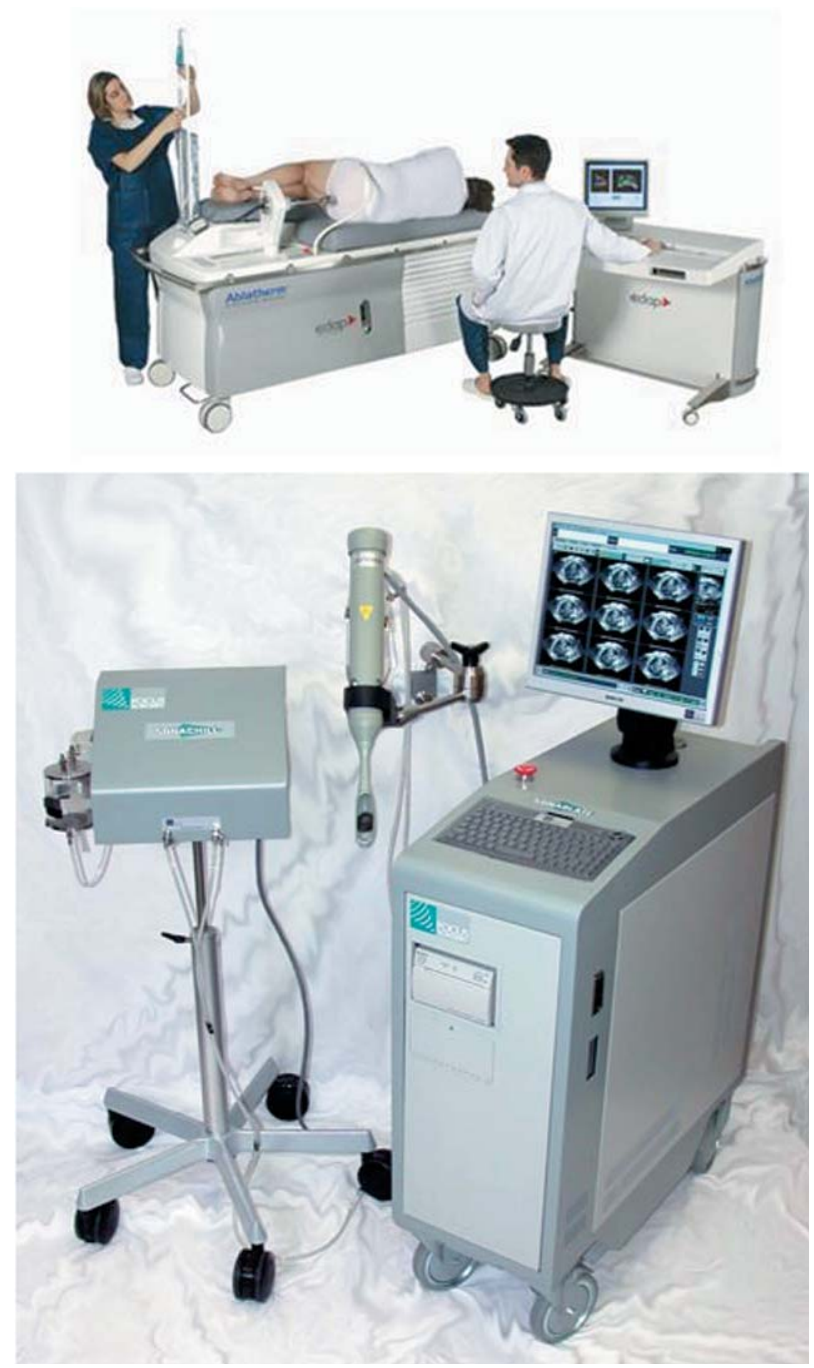

FIGURA 2. Dispositivos Ablatherm (EDAP) y Sonablate 500.

10,9 $\pm 8,7 \mathrm{ng} / \mathrm{ml}(<10: 50,9 \% ; 10-20: 3 \% ;>20: 16 \%)$. El 92\% de los pacientes habían mostrado 1-4 cilindros positivos en la biopsia diagnóstica. El Gleason medio fue de 2 -4 en $13,2 \%$, 5-7 en $77,5 \%$ y 8-10 en 9,3\%. Durante el seguimiento, se repitió la biopsia y el PSA. Cualquier patología positiva para Cap tras la última sesión de HIFU clasificó al paciente como fracaso terapéutico. Los pacientes recibieron una media de 1,4 sesiones de HIFU. El seguimiento medio fue de 407 días (cuartiles 1135-3598, media 321 días). El porcentaje de biopsias negativas observado en la población tratada fue de $87,2 \%$. El PSA tras tratamientos completados fue de $0,1 \mathrm{ng} / \mathrm{ml}$. Estos resultados fueron estratificados según el riesgo de la enfermedad, resultando en porcentajes de biopsia negativa de

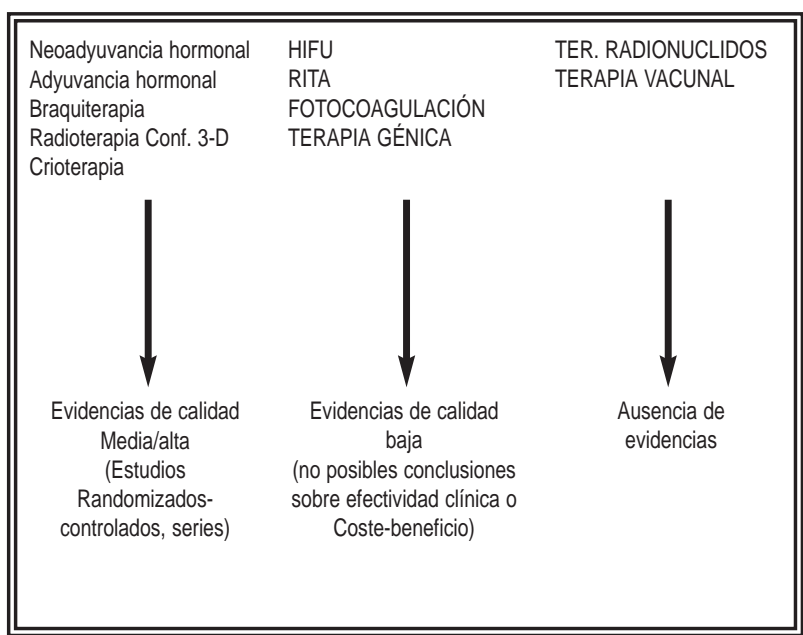

FIGURA 3. Evidencia científica de utilización de diversas opciones terapéuticas en cáncer prostático.

82-92\% (Tabla 1). El estudio concluye que HIFU debe ser considerada como una opción de tratamiento primario del Cap localizado, con un alto poder destructivo tumoral local. Las limitaciones de este trabajo, aunque la población de pacientes es considerable, son que el seguimiento resulta corto y que el ensayo no es randomizado. Además, no queda clara la interpretación de los valores de PSA en el seguimiento a medio plazo de los pacientes. No se refieren claramente la incidencia de complicaciones por la técnica.

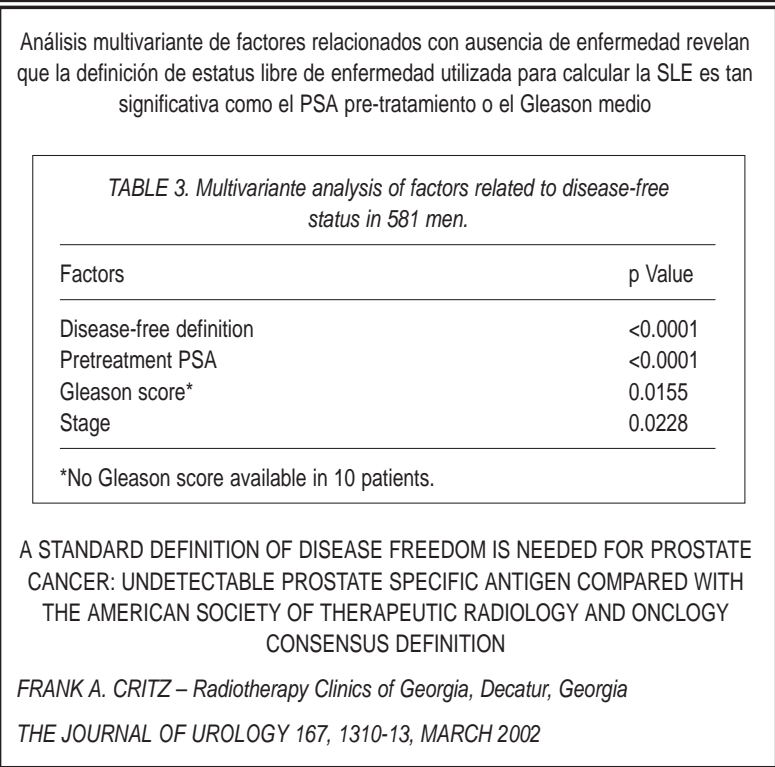

FIGURA 4. Importancia de la definición del estatus libre de enfermedad en el porcentaje de supervivencia libre de enfermedad. 
Tabla 1. Porcentaje de biopsias negativas tras HIFU según riesgo relacionado del adenocarcinoma prostático.

\begin{tabular}{lc}
\hline Riesgo relacionado Cap & $\begin{array}{c}\text { Porcentaje biopsias } \\
\text { negativas }\end{array}$ \\
\hline Pacientes bajo riesgo & $\mathbf{9 2 , 1}$ \\
T1-T2a y PSA $\leq 10 \mathrm{ng} / \mathrm{ml}$ y & \\
Gleason score $\leq 6$ & \\
Pacientes riesgo intermedio & $\mathbf{8 6 , 4 \%}$ \\
T2b o PSA $10,1-20 \mathrm{ng} / \mathrm{ml}$ o \\
Gleason score $=7$ \\
$\begin{array}{l}\text { Pacientes alto riesgo } \\
\text { T2c o PSA }>20 \mathrm{ng} / \mathrm{ml} \text { o } \\
\text { Gleason score } \geq 8\end{array}$ \\
\hline
\end{tabular}

El segundo trabajo seleccionado es una serie descriptiva de casos, provenientes de un solo centro. Ha sido publicado por Blana ${ }^{1}$. En él se analizan los efectos de HIFU en una serie de 146 pacientes Cap T1-T2 NO MO. Los criterios de inclusión fueron: diagnóstico patológico mediante biopsia transrectal, PSA de $</=15$ $\mathrm{ng} / \mathrm{ml}$ y Gleason $</=7$. Los individuos no fueron candidatos a prostatectomía radical, bien por comorbilidad o por no aceptar la intervención. El seguimiento medio fue de 22,5 meses (4-62) e incluyó determinaciones de PSA y biopsias sextantes de control. En cuanto a los resultados obtenidos, el PSA nadir determinado 3 meses tras el tratamiento fue de $0,07 \mathrm{ng} / \mathrm{ml}(0-$ $5,67 \mathrm{ng} / \mathrm{ml}$ ). El nivel medio de PSA después de un seguimiento de 22 meses fue de $0,15 \mathrm{ng} / \mathrm{ml}$ (0-12,11 ng/ml). Un 87\% de los pacientes mantuvieron unos valores de PSA constantes, inferiores a $1 \mathrm{ng} / \mathrm{ml}$. El $93,4 \%$ de los pacientes tuvieron biopsias negativas en los controles de seguimiento. Se refiere como complicación una fístula recto-uretral después de un retratamiento con HIFU en un paciente con antecedentes de hemicolectomía y fístulas anales recidivantes. El $12 \%$ de los pacientes fueron sometidos a resección transuretral prostática tras el HIFU por obstrucción urinaria, aunque los autores refieren ausencia completa de casos de incontinencia urinaria importante (grados 2-3). El $47,3 \%$ de los pacientes reconocen mantenimiento de la función eréctil. Finalmente, los autores no objetivan empeoramiento en el Indice de Calidad de Vida.
Blana concluye que estos resultados demuestran la eficacia y baja morbilidad asociada del HIFU. Este grupo plantea las teóricas y potenciales ventajas de la técnica como tratamiento inicial en los casos de Cap no candidatos a prostatectomía radical. Nuevamente se evidencia el poder destructivo tumoral local de la técnica $(>90 \%$ de biopsias de control negativas). Estos autores aplican 2 criterios distintos de SLE. Cuando utilizan el publicado por Amling en Journal of Urology en $2001^{22}$ (punto de corte de 0,4 ngrs/ml), obtienen una SLE de $71,5 \%$ a 35 meses. Sin embargo, cuando el punto de corte aplicado es el utilizado por Critz en $2002^{18}$, la SLE en el mismo período cae a $54 \%$.

¿Cuál es el mejor criterio de seguimiento en ausencia de clínica y/o biopsia positiva? El criterio para definir la recidiva bioquímica de la Asociación Americana de Radiología y Oncología (ASTRO) (3 incrementos consecutivos de PSA tras el tratamiento radical), publicado en $1997^{23}$, ha sido puesto en duda por Horwitz ${ }^{24}$ quien ha planteado que sobrestima la SLE comparativamente a otros criterios. Como hemos referido anteriormente, son múltiples los puntos de corte de PSA publicados en las distintas series de la literatura en los últimos años para explicar el concepto de SLE. Esta es una de las cuestiones actuales más controvertidas en el seguimiento del Cap tras tratamientos radicales. Ello es especialmente cierto si tenemos en cuenta que, de los pacientes con recidiva bioquímica tras cirugía radical ${ }^{21}$, sólo el $4 \%$ murieron de forma directamente relacionada con el tumor. Cuando el tratamiento aplicado había sido la radioterapia ${ }^{21}$, más de $10 \%$ de muertes en los pacientes con progresión bioquímica fueron atribuibles directamente a la neoplasia. El valor clínico de la progresión bioquímica es ciertamente confuso. Sin embargo, constituye un punto final (end-point) adecuado para los ensayos clínicos. La historia natural de la progresión bioquímica puede ser realmente larga. El PSA nadir puede ser un factor pronóstico importante para la evolución del paciente, determinado especialmente a los 3 meses del procedimiento; sin embargo ningún valor constituye un criterio absoluto para justificar el inicio de un tratamiento adyuvante. La realización de biopsias de control es completamente necesaria para verificar la 
eficacia local del tratamiento, e indicar si es necesario llevar a cabo un nuevo tratamiento local ${ }^{25}$.

Es difícil interpretar el valor real de las terapias emergentes, especialmente aquéllas que cuentan con seguimientos limitados en el tiempo. Este es el caso del ultrasonido de alta intensidad. Sí parece indudable la capacidad de destrucción tumoral local. Chaussy y Thüroff han publicado dos trabajos que documentan este aspecto. Se trata de revisiones de series propias de casos, con unos criterios de inclusión muy estrictos, un seguimiento de más de 5 años y un número considerable de pacientes incluidos (más de 500). En el primero de estos artículos ${ }^{26}$ se evalúa la eficacia histopatológica de la técnica. Ésta puede resultar en un 90\% de porcentaje de biopsias negativas a 5 años en pacientes con enfermedad de bajo y mediano riesgo. Además en la enfermedad localmente avanzada y diseminada consiguen porcentajes actuariales a 5 años de biopsias negativas de $78 \%$ y $60 \%$. Esta importante capacidad destructiva tumoral parece depender según estos mismos autores del esquema terapéutico aplicado y de la dosis administrada de ultrasoni$\mathrm{do}^{27}$ : existe un efecto tisular relacionado directamente con la dosis en la coagulación transrectal de la próstata. Cuando la estrategia inicial intenta tratar toda la glándula (RTU prostática+HIFU completo) o tras dos sesiones consecutivas, se obtienen mejores resultados. El porcentaje de retratamientos, la estabilidad del PSA y el PSA nadir dependen directamente del esquema inicial de tratamiento (Tabla 2).

El segundo grupo de trabajos que analizamos se refieren a la indicación del HIFU como terapia de rescate tras radioterapia radical (RR). La RR puede resultar en curación de los pacientes afectados por Cap localizado en, aproximadamente, un tercio de los $\operatorname{casos}^{28}$. El 39\% de los pacientes tratados con $\mathrm{RR}^{29}$ presentarian recidiva bioquímica tras una media de 2.9 años desde el tratamiento y el $23 \%$ podrían fallecer unos 5 años tras la progresión del PSA. En los pacientes con RR, la estimación del porcentaje de muerte cáncerespecífica 5 años tras la progresión de PSA fue de $24 \%, 40 \%$ y $59 \%$ en casos con Gleason, respectivamente, de $</=6,3+4$ o $4+3$ u $8-10$ respectivamente. El planteamiento terapéutico en estos pacientes es bastante dispar. La prostatectomía radical de rescate puede conseguir porcentajes de SLE a 10 años aceptables $(43 \%)^{30}$. Sin embargo, la aparición de complicaciones secundarias es

Tabla 2. Porcentaje de biopsias negativas-PSA nadir medio-estabilidad de PSA-velocidad media de PSA y porcentaje de retratamiento tras terapia con Ultrasonido de Alta Intensidad en distintos esquemas terapéuticos en cáncer prostático

\begin{tabular}{|c|c|c|c|c|c|c|c|c|c|c|c|c|}
\hline \multirow[t]{2}{*}{$\mathrm{N}=430$} & \multirow{2}{*}{$\begin{array}{l}\text { HIFU } \\
\text { mono } \\
\text { A }\end{array}$} & \multirow[b]{2}{*}{ B } & \multicolumn{2}{|c|}{$\begin{array}{l}\text { TURP+ } \\
\text { HIFU }\end{array}$} & \multicolumn{2}{|c|}{ Partial } & \multicolumn{2}{|c|}{ Complete } & \multicolumn{2}{|l|}{$\begin{array}{l}\text { Nerve } \\
\text { sparing }\end{array}$} & \multicolumn{2}{|c|}{$\begin{array}{l}\text { No nerve- } \\
\text { sparing }\end{array}$} \\
\hline & & & & B & $\mathbf{A}$ & B & $\mathbf{A}$ & B & A & B & & B \\
\hline $\begin{array}{l}\text { Negative } \\
\text { biopsy } \\
\text { rate (\%) }\end{array}$ & 78,1 & 86,8 & 84,1 & 93,8 & 79,5 & 88,4 & 83,1 & 91,2 & 66,7 & 92,1 & 81,9 & 88,6 \\
\hline $\begin{array}{l}\text { Median } \\
\text { nadir PSA } \\
(\mathrm{ng} / \mathrm{ml})\end{array}$ & 0,2 & 0,0 & 0,0 & 0,0 & 0,2 & 0,1 & 0,0 & 0,0 & 1,2 & 0,0 & 0,0 & 0,0 \\
\hline $\begin{array}{l}\text { PSA } \\
\text { stability } \\
\text { rate (\%) }\end{array}$ & 78,1 & 81,4 & 82,9 & 82,4 & 78,5 & 81,6 & 82,8 & 81,8 & 75,0 & 83,3 & 80,0 & 81,6 \\
\hline $\begin{array}{l}\text { Median PSA } \\
\text { velocity } \\
\text { (ng/ml/y) }\end{array}$ & 0,66 & 0,27 & 0,15 & 0,03 & 0,62 & 0,29 & 0,15 & 0,0 & 0,91 & 0,34 & 0,35 & 0,12 \\
\hline $\begin{array}{l}\text { HIFU } \\
\text { retreatment } \\
\text { rate }(\%)\end{array}$ & 40,1 & & 7,7 & & 32,5 & & 9,4 & & 32,3 & & 21,8 & \\
\hline
\end{tabular}

HIFU mono: monoterapia HIFU, TURP+HIFU: RTU próstata+HIFU, partial: HIFU parcial, complete: HIFU completo, nerve parting: HIFU con respeto de fascículos neurovasculares; no nerve-sparing: HIFU sin respeto de FNV. De Chaussy C, Thuroff S, Kiel HF. Transrectal high-intensity focused ultrasouns for prostate cancer: impact of the energy dose. European Urology Supplements, 3 (2), February 2004 , page 213. 
alta, con una incidencia de incontinencia urinaria de más de $40 \%$. Otras técnicas utilizables serían la braquiterapia, con SLE de $34 \%$ a 5 años aunque con menos efectos secundarios que la cirugía $^{31}$. La crioterapia ofrece resultados controvertidos, que dependerían mucho del autor evaluado $^{32}$. No existe evidencia científica de calidad significativa sobre la mejor terapia de rescate en la recidiva local post-RR. La literatura carece de ensayos clínicos randomizados con series amplias que comparen técnicas con seguimientos largos.

En cuanto a la utilización del HIFU como terapia de rescate en recidivas locales post-RR destaca un trabajo de Gelet publicado en Urology ${ }^{20}$. Se trata de una serie de 71 casos, con una edad media de $67 \pm 5,86$ años. El volumen prostático medio transrectal pre-HIFU fue de $21,4 \pm 11,1 \mathrm{~cm} 3$. No hubo casos con diseminación a distancia objetivada previamente al HIFU. Todos los pacientes presentaban biopsia positiva. El 66,2\% de los casos presentaron Cap moderadamente/mal diferenciados. Se administraron 85 sesiones en 71 pacientes $(1,2$ sesiones/paciente). El seguimiento medio fue de 14,8 meses (6-86). El valor de PSA nadir determinado a 3 meses tras el tratamiento con HIFU fue de $1,97 \mathrm{ngrs} / \mathrm{ml}$.

En cuanto a los resultados patológicos el 80\% de los pacientes presentaron biopsias prostáticas de control negativas. Sin embargo, 40 casos (56\%) mostraron elevación de PSA sérico a lo largo del seguimiento. De este grupo sólo se objetivó mediante biopsia cáncer residual prostático en 14; los 26 restantes ofrecieron elevación aislada de PSA con biopsia prostática negativa. La SLE fue evaluada a 30 meses de dos formas: cuando únicamente se tenían en cuenta criterios histológicos era de $73 \%$. Cuando además se incluían criterios bioquímicos (progresión de PSA), ese valor se reducía al $37 \%$.

Las reflexiones que hacen los autores, a modo de conclusiones, plantean al ultrasonido de alta intensidad como una terapia de rescate útil en la recidiva local del Cap tras $\mathrm{RR}$, con unos porcentajes altos de control local de la enfermedad. Esta técnica ofrece una morbilidad aceptable comparativamente con otras alternativas. La limitación, sin embargo, de este trabajo, al igual que en series similares tratadas con otras técnicas, es la dificultad para diagnosticar el estadio tumoral real de estos pacientes, así como para conocer el significado clínico real a largo plazo de la progresión bioquímica y su influencia pronóstica cuando se utiliza para determinar la SLE.

\section{CONCLUSIONES}

A la vista de lo revisado en la Literatura, y apoyados por las reflexiones del Comité Oncológico de la Asociación Francesa de Urologia ${ }^{33}$ podemos plantear las siguiente conclusiones:

1. Existen cada vez más estudios que evalúan la eficacia y morbilidad del HIFU

2. Aunque los seguimientos aumentan, debemos valorar mejor la eficacia en términos de curación definitiva

3. Según el Estudio Multicéntrico Europeo ${ }^{8}$ HIFU podría ser una alternativa válida en el Cap bien y moderadamente diferenciado, con PSA $<15 \mathrm{ngrs} / \mathrm{ml}$ y esperanza de vida de 10-15 años.

4. En Cap con riesgo bajo/moderado, HIFU puede conseguir resultados similares a otras terapias. En tumores de alto riesgo, no puede aún considerarse como monoterapia (sí en tratamientos combinados o en el marco de ensayos clínicos randomizados)

5. Como terapia de rescate, HIFU ofrece resultados prometedores. Sus ventajas: baja morbilidad, posibilidad de retratamiento, radioterapia administrable tras fracaso.

\section{REFERENCIAS}

1. Blana A, Walter B, Rogenhofer S, Wieland WF. High-intensity focused ultrasound for the treatment of localized prostate cancer: 5-year experience. Urology. 2004;63(2):297300 .

2. Hummel S, Paisley S, Morgan A, Curie E, Brewer N. Clinical and cost-effectiveness of new and emerging technologies for early localised prostate cancer: a systematic review. Health Technol Assess. 2003;7(33):iii, ix-x, 1-157.

3. Herranz Amo F, Arias Funez F, Arrizabalaga Moreno M, Calahorra Fernández FJ, Carballido Rodríguez J, Diz Rodríguez R, et al. El cáncer de próstata en la Comunidad de Madrid en el año 2000. I-Incidencia. Actas Urol. Esp. 2003;27(5):323-334.

4. Huber P, Debus J, Jenne J, Jöchle K, van Kaick G, Lorenz WJ, et al. Therapeutic ultrasound in tumor therapy: principles, applications and new developments. Radiologe. 1996;36(1):64-71.

5. Chapelon JY, Margonari J, Vernier F, Gorry F, Ecochard R, Gelet A. In-vivo effects of high-intensity ultrasound on prostatic adenocarcinoma Dunning R3327. Cancer Res.1996;52 (22):6353-6357. 
6. Chaussy CG, Thüroff S. High-Intensity focused ultrasound in localized prostate cancer. J Endourol. 2000; 14:293-299.

7. Chaussy C, Thürof S, Rebillard X, Gelet A. Technology insight: high-intensity focused ultrasound for urologic cancers. Nat Clin Pract Urol. 2005;2(4):191-198.

8. Ter Haar G. Intervention and therapy. Ultrasound Med Biol. 2000;26 Suppl 1:51-54.

9. Chapelon JY, Margonari J, Theillère Y, Gorry F, Vernier F, Blanc E, et al. Effects of high-energy focused ultrasound on kidney tissue in the rat and the dog. Eur Urol. 1992;22(2): 147-152.

10. Kishi M. Expermiental studies of effects of intense ultrasound on implantable murine glioma. In: Proceedings of the 2nd European Congress on Ultrasonics in Medicine: 28-33 (Eds Kazmer E et al). Amsterdam, Excerpta Medica 1975.

11. Moore WE, Lopez RM, Matthews DE, Sheets PW, Etchison MR, Hurwitz AS, et al. Evaluation of high-intensity therapeutic ultrasound irradiation in the treatment of experimental hepatoma. J Pediatr Surg. 1989;24(1):30-33;discussion 33 .

12. Yang R. High-intensity focused ultrasound on prostatic adenocarcinoma Dunning R3327. Cancer Res. 1991;52: 6353-6357.

13. Oosterhof GO, Cornel EB, Smits GA, Debruyne FM, Schalken JA. In vivo effects of high-intensity focused ultrasound on the development of metastases. Eur Urol. 1997;32(1):91-95.

14. Damianou C. In vitro and in vivo ablation of porcine renal tissues using high-intensity focused ultrasound. Ultrasound Med Biol. 2003;29(9):1321-1330.

15. Gelet A, Chapelon JY, Margonari J, Theillere Y, Gorry F, Cathignol D, et al. Prostatic tissue destruction by highintensity focused ultrasound: experimentation on canine prostate. J Endourol. 1993;7(3):249-253.

16. Vallancien G, Chartier-Kastler E, Harouni M, Chopin D, Bougaran J. Focused extracorporeal pyrotherapy: experimental study and feasibility in man. Semin Urol. 1993; 11(1):7-9.

17. Wu F, Wang ZB, Chen WZ, Bai J, Zhu H, Qiao TY. Preliminary experience using high-intensity focused ultrasound for the treatment of patients with advanced stage renal malignancy. J Urol. 2003;170(6 Pt 1):2237-2240.

18. Critz FA. A standard definition of disease freedom is needed for prostate cancer: undetectable prostate specific antigen compared with the American Society of Therapeutic Radiology and Oncology consensus definition. J Urol. 2002;167(3):1310-1313.

19. Thüroff S, Chaussy C, Vallancien G, Wieland W, Kil HJ, Le Duc A, et al. High-Intensity Focused Ultrasound and Localized Prostate Cancer: Efficacy Results from the European Multicentric Study. J Endourol. 2003;17(8):673677.

20. Gelet A, Chapelon JY, Poisonier L, Bouvier R, Rouvière O, Curiel L, et al. Local recurrence of prostate cancer after external beam radiotherapy: early experience of salvage therapy using high-intensity focused ultrasonography. Urology.2004;63(4):625-629.
21. Moul JW. Variables in predicting survival based on treating "PSA-only" relapse. Urol Oncol. 2003;21(4):292-304.

22. Amling CL, Bergstralh EJ, Blute ML, Slezak JM, Zincke H. Defining prostate specific antigen pogresion after radical prostatectomy: what is the most appropiate cut point?. J Urol. 2001;165(4):1146-1151.

23. Consensus statement: guidelines for PSA following radiation therapy. American Society for Therapeutic Radiology and Oncology Consensus Panel. Int J Radiat Oncol Biol Phys. 1997;1537(5):1035-1041.

24. Horwitz EM, Uzzo RG, Hanlon AL, Greenberg RE, Hanks GE, Pollack A. Modifying the American Society fo therapeutic radiology and oncology definition of biochemical failure to minimize the influence of backdating in patients with prostate cancer treated with 3-dimensional conformal radiation therapy alone. J Urol. 2003;169(6):2153-7; discussion 2157-2159.

25. Gelet A, Chapelon JY, Bouvier R, Rouviere O, Poissonier L, Pasticier G, et al. Transrectal focused ultrasound and localized prostate cancer: may the nadir PSA predict the treatment success?. European Urology Supplements. 2004; $3(2): 213$

26. Thüroff S, Kiel HJ, Chausy C. Local histopathological efficacy of HIFU. European Urology Supplements 3(2): 213, 2004.

27. Chaussy C, Thüroff S, Kiel HJ. Transrectal High Intensity Focused Ultrasound for Prostate Cancer: impact of the energy dose. European Urology Supplements. 3(2):213, 2004.

28. Stephenson RA. Population-based prostate cancer trends in the PSA-era: data from the surveillance, epidemiology and end results program. Mongr Urol. 19:1-19,1998.

29. D’Amico AV, Cote K, Loffredo M, Renshaw AA, Chen MH. Pretreatment predictors of time to cancer specific death after prostate specific antigen failure. J Urol. 2003;169(4): 1320-1324.

30. Amling CL, Lerner SE, Martin SK, Slezak JM, Blute ML, Zincke H. Deoxyribonucleic acid ploidy and serum prostate specific antigen predict outcome following salvage prostatectomy for radiation refractory prostate cancer. J Urol. 1999;161(3):857-62; discussion 862-863.

31. Grado GL, Collins JM, Kriegshauser JS. Salvage brachytherapy for localized prostate cancer after radiotherapy failure. Urology. 1999;53(1):2-10.

32. Pisters LL, von Eschenbach AC, Scott SM, Swanson DA, Dinney CP, Pettaway CA, et al. The efficacy and complications of salvage criotherapy of the prostate. J Urol. 1997; 157(3):921-925.

33. Rebillard X, Davin JL, Soulie M, Comité de Cancerologie de 1’Associacion Francaise d'Urologie. Progr Urol. 2003;13(6): 1428-1456.

Correspondencia autor: Dr. E. Lledó García

Servicio de Urología. Hospital General Univ. Gregorio Marañón

Doctor Esquerdo, 46 - 28007 Madrid.

Tel.: 915868000

E-mail: ENLLGA@terra.es

Información artículo: Original 